\title{
Metodología de diseño para robots paralelos de cinco eslabones y dos grados de libertad
}

\author{
Design Methodology for five-bar Parallel Robots with two Degrees of Freedom
}

\section{David González-Reyes ${ }^{1}$, Heebum Kim², Daniela Rubio-Martínez ${ }^{3}$ Héctor Cervantes-Culebro ${ }^{4}$, Milton Elías-Espinosa ${ }^{5}$}

\author{
1 Instituto Tecnológico y de Estudios Superiores de Monterrey, MÉXICO \\ https://orcid.org/0000-0002-1535-3007 | dgr@exatec.tec.mx \\ 2Instituto Tecnológico y de Estudios Superiores de Monterrey, MÉXICO \\ https://orcid.org/0000-0002-2330-3026 | Heebum.Kim@exatec.tec.mx \\ 3Instituto Tecnológico y de Estudios Superiores de Monterrey, MÉxICO \\ https://orcid.org/0000-0003-1221-5431 | a01165714@exatec.tec.mx \\ 4 Instituto Tecnológico y de Estudios Superiores de Monterrey, MÉXICO \\ https://orcid.org/0000-0002-5481-2640 | hector_cervantes@tec.mx \\ 5 Instituto Tecnológico y de Estudios Superiores de Monterrey, MÉXICO \\ https://orcid.org/0000-0002-1377-3569 | mielias@tec.mx
}

Recibido 05-10-2020, aceptado 19-12-2020.

\section{Resumen}

En el presente trabajo se propone una metodología de diseño de robots paralelos de cinco eslabones y dos grados de libertad que hace uso de herramientas tecnológicas para simplificar el proceso de diseño. El uso de simuladores permite el diseño sin necesidad del uso y cálculo del modelo dinámico del sistema, de esta manera agilizar el proceso de diseño y permitir ajustes en el diseño de manera eficiente. La metodología se presenta con un diagrama de flujo donde se desarrollan los siguientes pasos: definición de problema y restricciones, cálculo de cinemática inversa, propuesta de longitud de eslabones, propuesta de actuadores, propuesta de diseño y material de eslabones, diseño $\mathrm{CAD}$, simulación dinámica, análisis por elemento finito, construcción de prototipo, programación de microcontrolador, selección de etapa de potencia y diseño de interfaz. Un robot paralelo de cinco eslabones y dos grados de libertad es desarrollado para ejemplificar la metodología de diseño y se valida que este cumpla adecuadamente con el problema y la tarea definida.

Palabras clave: Cinemática, diseño, metodología, simulación, motor brushless.

\begin{abstract}
In this paper, a methodology for the design of two-degree-of-freedom five-bar parallel robots is proposed that makes use of technological tools to simplify the design process. The use of simulators allows design without the need for the use and calculation of the dynamic model of the system, thus speeding up the design process and allowing design adjustments efficiently. The methodology is presented with a flow diagram where the following steps are developed: definition of problem and constraints, calculation of inverse kinematics, proposal of the length of the links, proposal of actuators, proposal of design and material of links, CAD design, dynamic simulation, finite element analysis, prototype construction, microcontroller programming, power stage selection and interface design. A two-degree-of-freedom five-bar robot is developed to exemplify the design methodology and it is validated that it fulfills the problem and the defined task.
\end{abstract}

Index terms: Brushless motor, design methodology, kinematics, simulation. 


\section{INTRODUCCIÓN}

En la literatura se pueden encontrar múltiples metodologías para el diseño de sistemas mecatrónicos, como: método óptimo robusto, que se enfoca en un diseño menos sensible ante variables de incertidumbre, proponiendo índices de firmeza [1]. Otra metodología es diseño óptimo de la fiabilidad, que busca la optimización ante externalidades por medio de probabilidades de fallo y de valores esperados [1]. La metodología de diseño concurrente conjunta el diseño de la estructura mecánica y el control, de manera paralela, con el fin de optimizar el proceso de generación de producto [2]. El método paramétrico robusto busca maneras de controlar la robustez ante factores externos llamados ruido, como factores ambientales, condiciones de temperatura, o desgaste del mecanismo [3].

Las metodologías presentadas anteriormente necesitan conocer el modelo dinámico para el diseño de robots. Sin embargo, para la metodología de diseño de un robot paralelo de cinco eslabones y dos grados de libertad, que se estudia en este trabajo, no es necesario conocer el modelo dinámico del robot. Esto se debe a que se utiliza la síntesis estructural y la dinámica no lineal del sistema para la propuesta de actuadores. La metodología de diseño presente propone un procedimiento para construir un robot de dos grados de libertad.

El modelado dinámico de un robot necesita la cinemática inversa del robot paralelo de cinco eslabones para ser resuelta. Los diferentes métodos permiten generar el modelo dinámico y cinemático del robot a partir de métodos numéricos que relacionan fuerzas, velocidades, energía, posiciones o pares [4]. Entre los modelos dinámicos más comunes para el robot paralelo se encuentran el Método de Euler- Lagrange, Método Newton Euler [5], Método de Rigidez dinámica y el Método de Gibbs-Appell.

El modelo dinámico a partir del método de Newton sería mayormente útil para una cadena cinemática sin demasiadas restricciones. En el método de rigidez dinámica se debe establecer la cadena cinemática compuesta por cuerpos rígidos y flexibles que se conectan por articulaciones. La matriz de rigidez mapea las deformaciones de los nodos que contienen fuerzas dinámicas y los pares [6]. Para los robots paralelos se deben incluir los operadores de Lagrange para cumplir las restricciones de cadena cinemática cerrada. El Método Gibbs-Appell analiza el sistema a partir de la transformación de un sistema restringido de ecuaciones de número de coordenadas generalizadas y de ecuaciones de restricción en un sistema de ecuaciones diferenciales ordinarias. Esto se logra mediante la separación de varias cadenas abiertas que se obtienen del sistema mecánico restringido [7]. Por lo tanto, existe una complejidad en resolver la restricción de cadena cinemática usando los métodos de modelado mencionados anteriormente.

El presente trabajo busca eliminar este modelado dinámico, principalmente porque es complicado el análisis de dos cadenas cinemáticas donde una de la otra para determinar la posición del efector final. Las simulaciones evitan que el diseñador tenga que resolver las restricciones de cadena cinemática cerrada en el modelo dinámico con el fin de agilizar el proceso de diseño y que posibles o ajustes cambios sean fáciles de realizar.

\section{Desarrollo}

La metodología de diseño propuesta se ejemplifica con el diagrama de flujo de la Fig. 1. Posteriormente, se encuentra el desarrollo de cada paso del proceso descrito en el diagrama. 
$m$
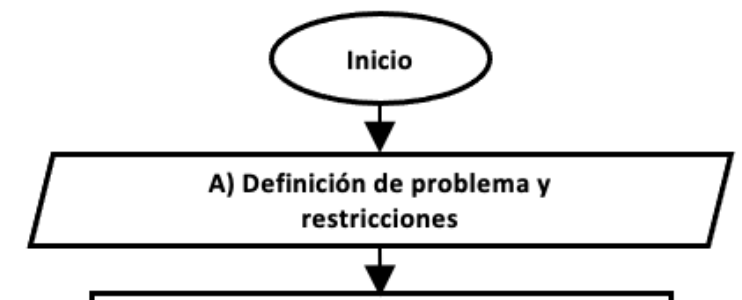

B) Cálculo de cinemática inversa

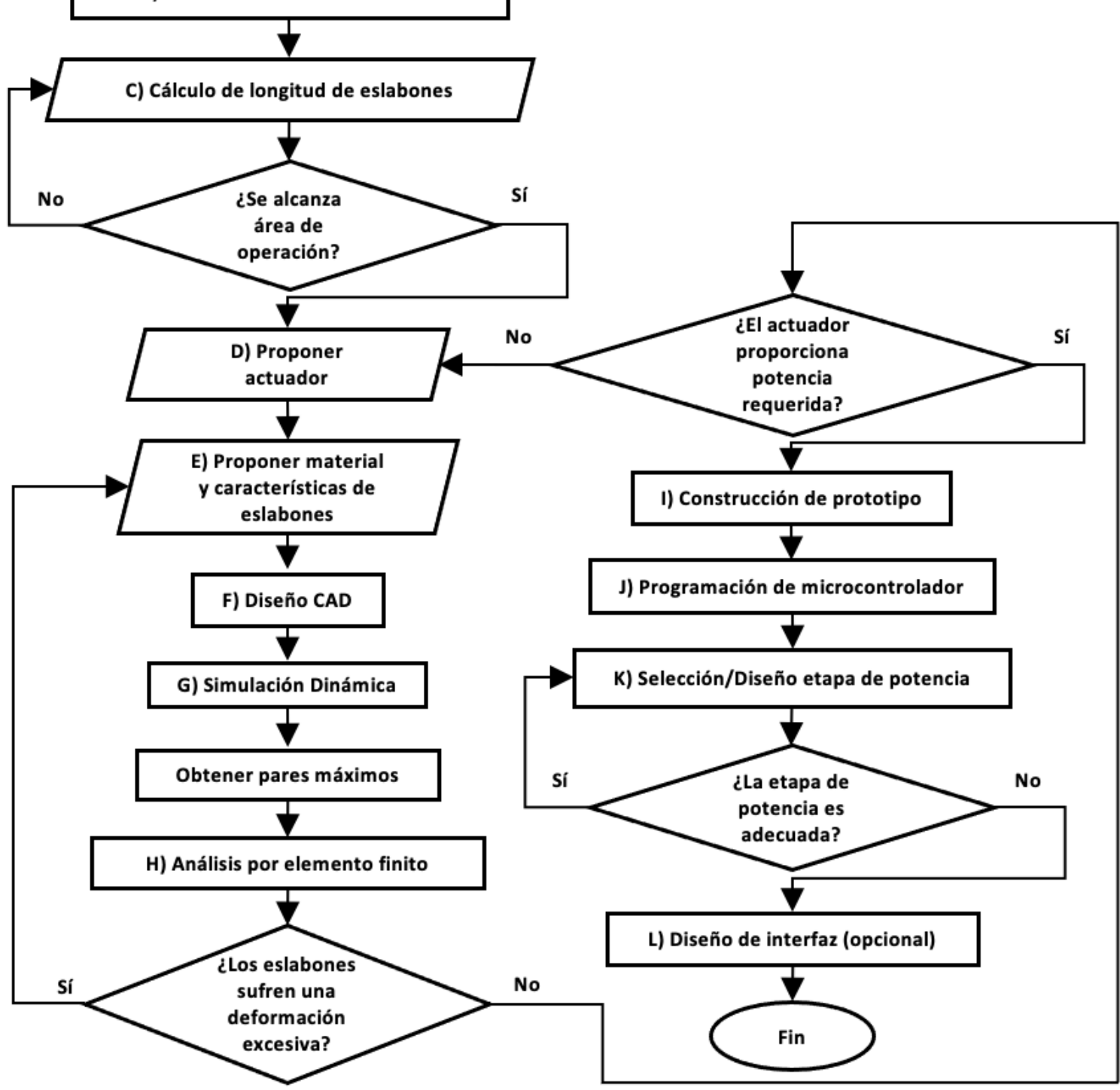

Fig. 1. Diagrama de flujo de la metodología propuesta. 


\section{A. Definición del problema y restricciones}

El primer paso del diseñador es definir la tarea que desea llevar a cabo el robot a diseñar, en qué tiempo y con qué precisión. La aplicación más común del robot de cinco barras paralelo es para tareas de pick and place. Aquí se definen los puntos en el plano que el robot de dos grados de libertad debe alcanzar, delimitando un área de operación y se planea una trayectoria. Posteriormente, se deben marcar las restricciones como son: capacidad máxima de carga, tipo de efector final, presupuesto, materiales, acceso a equipo y herramienta, entre otras.

En el presente se ejemplifica la metodología de diseño propuesta al desarrollar un robot paralelo de cinco eslabones y dos grados de libertad. Dicho robot busca mover piezas por debajo de $100 \mathrm{~g}$ al menos $25 \mathrm{~cm}$ paralelos a la línea que pasa por el centro de rotación de los actuadores, con una velocidad mínima de $0.5 \mathrm{~m} / \mathrm{s}$ y una precisión de $\pm 5 \mathrm{~mm}$.

\section{B. Cálculo de cinemática inversa}

El análisis de cinemática inversa permite conocer las posiciones angulares de los actuadores al proponer una posición del efector final en el plano $(x, y)$. Para el robot paralelo de cinco eslabones, se utiliza trigonometría para su cálculo. La Fig. 2 le asigna un nombre a cada eslabón del robot.

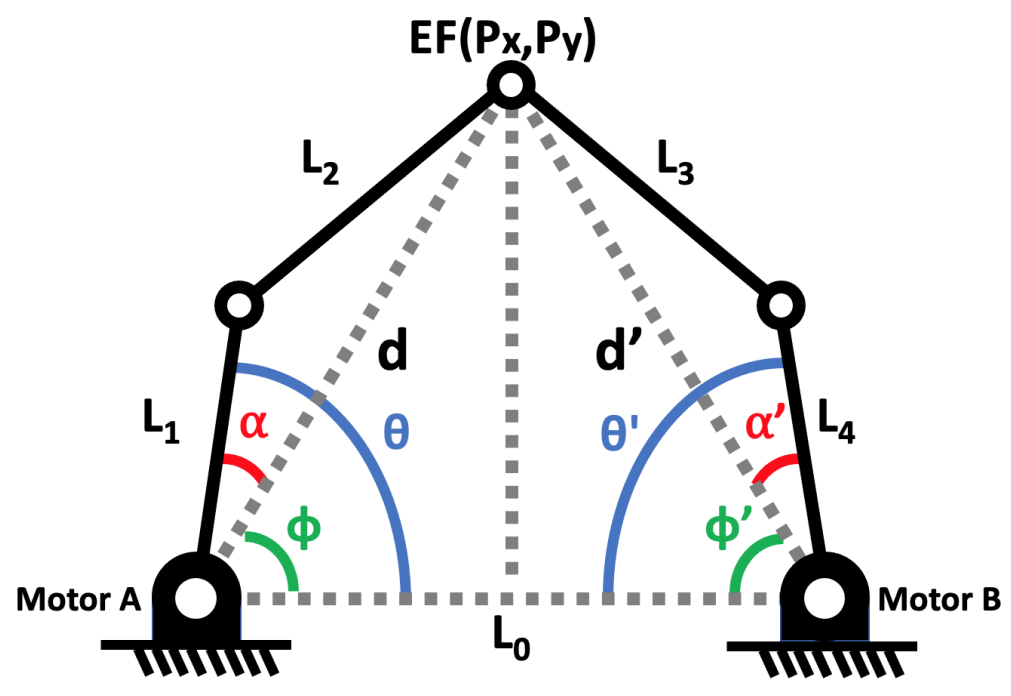

Fig. 2. Modelo matemático.

$$
\phi=\operatorname{atan}(P y / P x)
$$

Conociendo el ángulo $\phi$ a partir de (1):

$$
\begin{gathered}
d=\sqrt{P x^{2}+P y^{2}} \\
L_{2}^{2}=d^{2}+L_{1}{ }^{2}-2 * d * L_{1} \cos (\alpha)
\end{gathered}
$$




$$
\alpha=\operatorname{acos}\left(\frac{d^{2}+L_{1}{ }^{2}-L_{2}{ }^{2}}{2 * d * L_{1}}\right)
$$

Posición angular de actuador izquierdo:

$$
\theta=\alpha+\phi
$$

10

Del lado derecho se realiza el mismo análisis, sin embargo, la distancia $P x$ es reemplazada por $L_{0}-P x$ para obtener los ángulos deseados.

$$
\begin{gathered}
\phi^{\prime}=a \tan \left(\frac{P y}{L_{0}-P x}\right) \\
d^{\prime}=\sqrt{\left(L_{0}-P x\right)^{2}+P y^{2}} \\
\alpha^{\prime}=a \cos \left(\frac{d^{\prime 2}+L_{4}^{2}-L_{3}^{2}}{2 * d^{\prime} * L_{4}}\right)
\end{gathered}
$$

Posición angular de actuador derecho:

$$
\theta^{\prime}=\alpha^{\prime}+\phi^{\prime}
$$

\section{C) Cálculo de longitud de eslabones}

El área de trabajo de un robot se da por las longitudes de sus eslabones. El cálculo del área de trabajo de un robot de cinco barras paralelo se puede simplificar a dos ecuaciones si se consideran las restricciones (10), (11), (12) y (13).

$$
\begin{gathered}
L_{1}=L_{4} \\
L_{2}=L_{3} \\
L_{0}<L_{1}+L_{2}+L_{3}+L_{4} \\
L_{0}+L_{1}+L_{4}=L_{2}+L_{3}
\end{gathered}
$$

Tomando en cuenta estas restricciones, el área de trabajo se calcula a partir de la intersección de las áreas dadas por la ecuación (14) restando las áreas dadas por la ecuación (15), [8]. El usuario debe proponer longitudes para los eslabones y calcular el área de trabajo hasta que se valide que la trayectoria propuesta (sección A) se encuentra dentro de esta.

$$
\left(P_{x}+L_{0}\right)^{2}+P_{y}^{2}=\left(L_{1} \pm L_{2}\right)^{2}
$$




$$
\left(P_{x}-L_{0}\right)^{2}+P_{y}^{2}=\left(L_{1} \pm L_{2}\right)^{2}
$$

Para encontrar las posibles soluciones que cumplan con la tarea definida en la sección A se desarrolla un script (programa) en Matlab que calcula todas las áreas de trabajo $\left(\mathrm{en}^{\mathrm{mm}^{2}}\right.$ ) posibles al insertar un límite superior y un límite inferior a la longitud de los cinco eslabones. Estos límites pueden provenir por límites físicos, por un espacio de instalación, límites del proceso de manufactura, límites a partir de peso, acceso a material, etc. Posteriormente, se buscan las dimensiones que maximicen el área de trabajo. El procedimiento del script está descrito en el diagrama de flujo de la Fig. 3. El script necesita que se proponga un área de trabajo meta donde se escoge un rectángulo de $400 \times 800 \mathrm{~mm}\left(480,000 \mathrm{~mm}^{2}\right)$. Este calcula el porcentaje del área de trabajo que puede cubrir el robot con respecto al área propuesta y guarda el resultado de cada iteración en un archivo en forma de lista. Para reducir el número de iteraciones del ciclo del script y el tiempo de cálculo se indica que las longitudes $L_{1}, L_{2}, L_{3}$ y $L_{4}$ aumenten de $5 \mathrm{~mm}$ en $5 \mathrm{~mm}$.

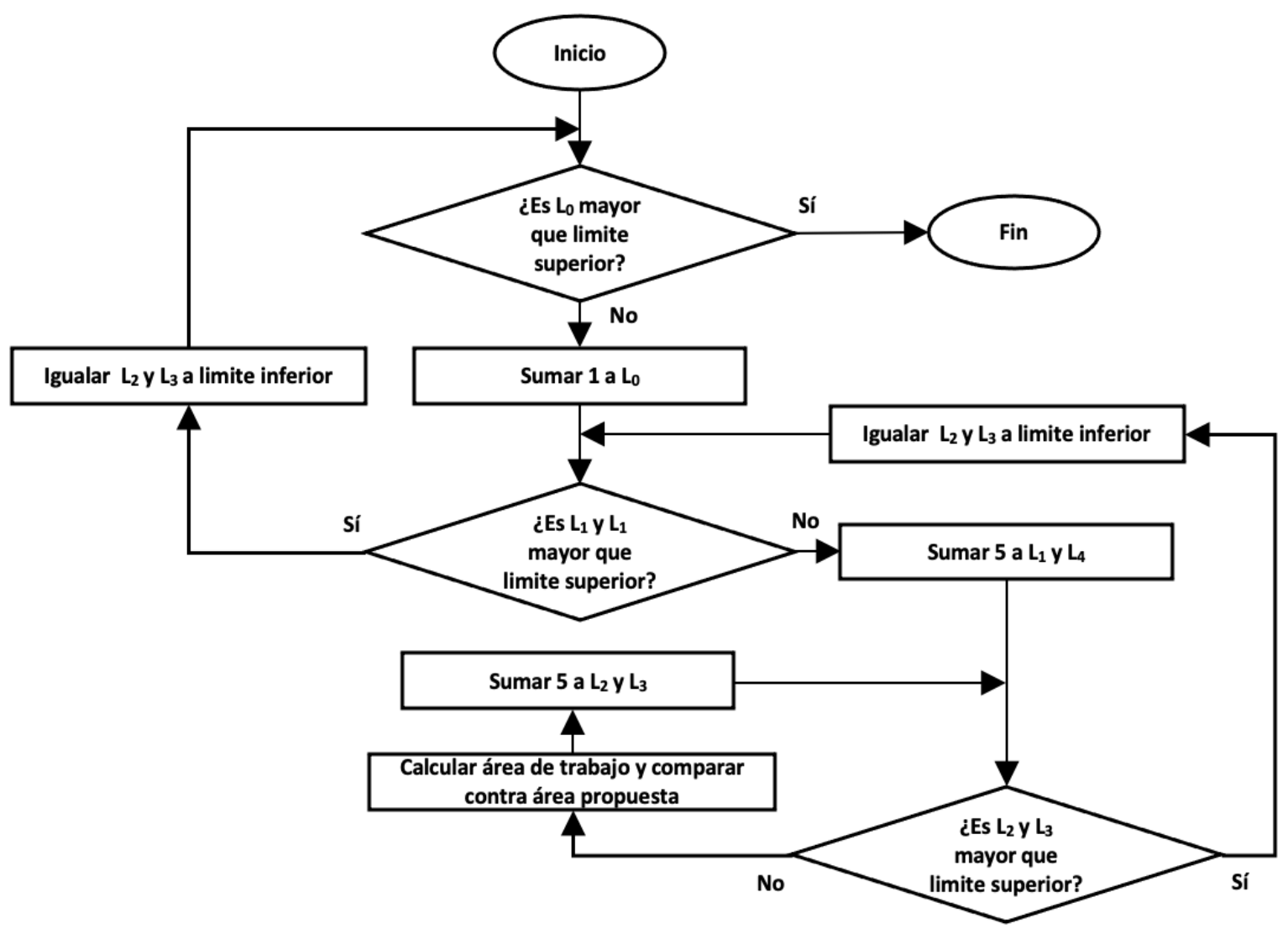

Fig. 3. Diagrama de flujo del script de Matlab

Una vez que el programa es ejecutado, se busca entre las más de 30000, iteraciones las combinaciones de eslabones que cumplen con la restricción mostrada en la ecuación (5) y dentro de estas se buscan las combinaciones que maximizan el área de trabajo. Al graficar las longitudes de los eslabones con respecto al área total de trabajo, como se muestra en las Figs. 4, 5, y 6, se observa la tendencia que al aumentar la longitud de $L_{0}$ disminuye el área de trabajo mientras que al aumentar la longitud del resto de los eslabones el área de trabajo total aumenta. 


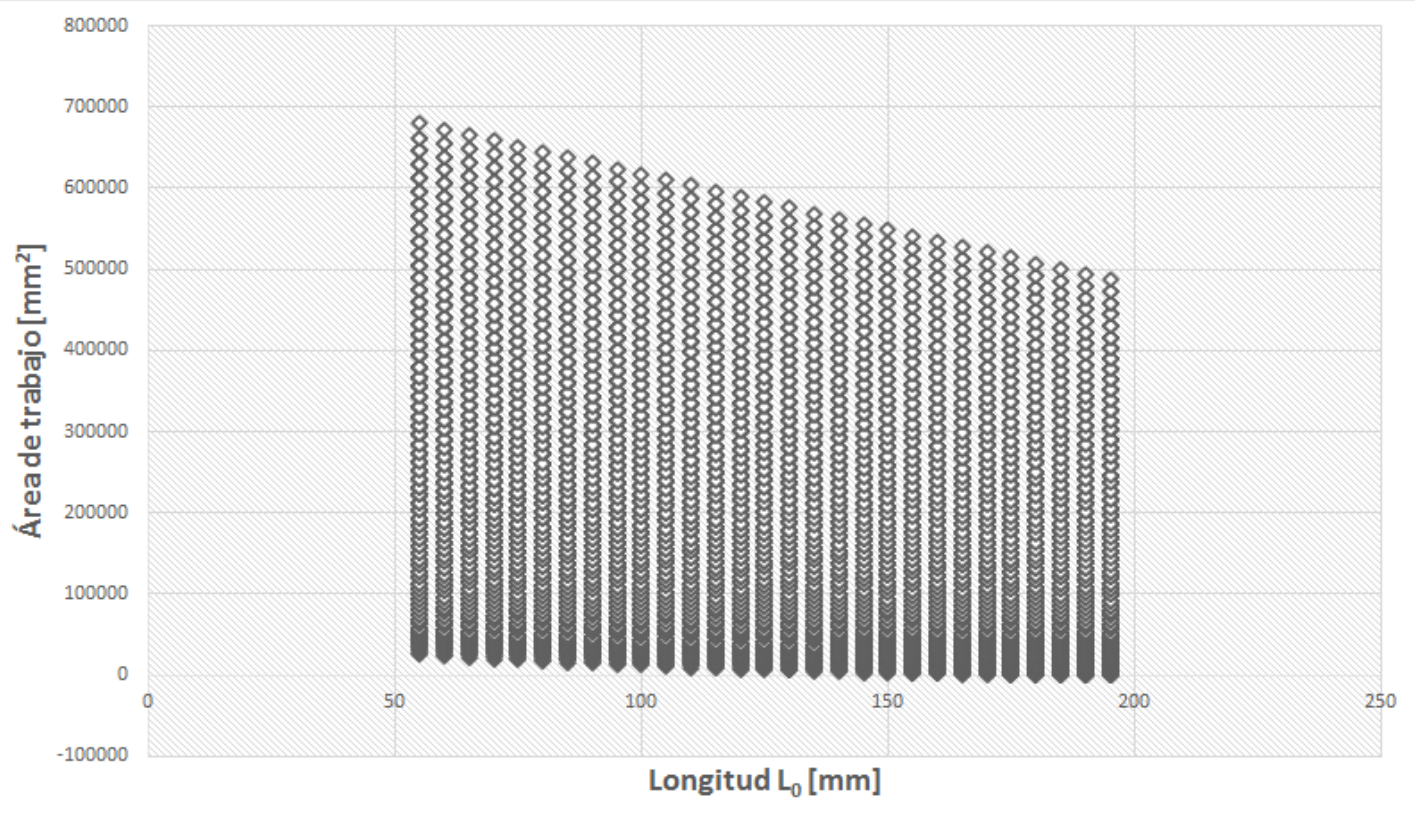

Fig. 4. Lo vs. Área.

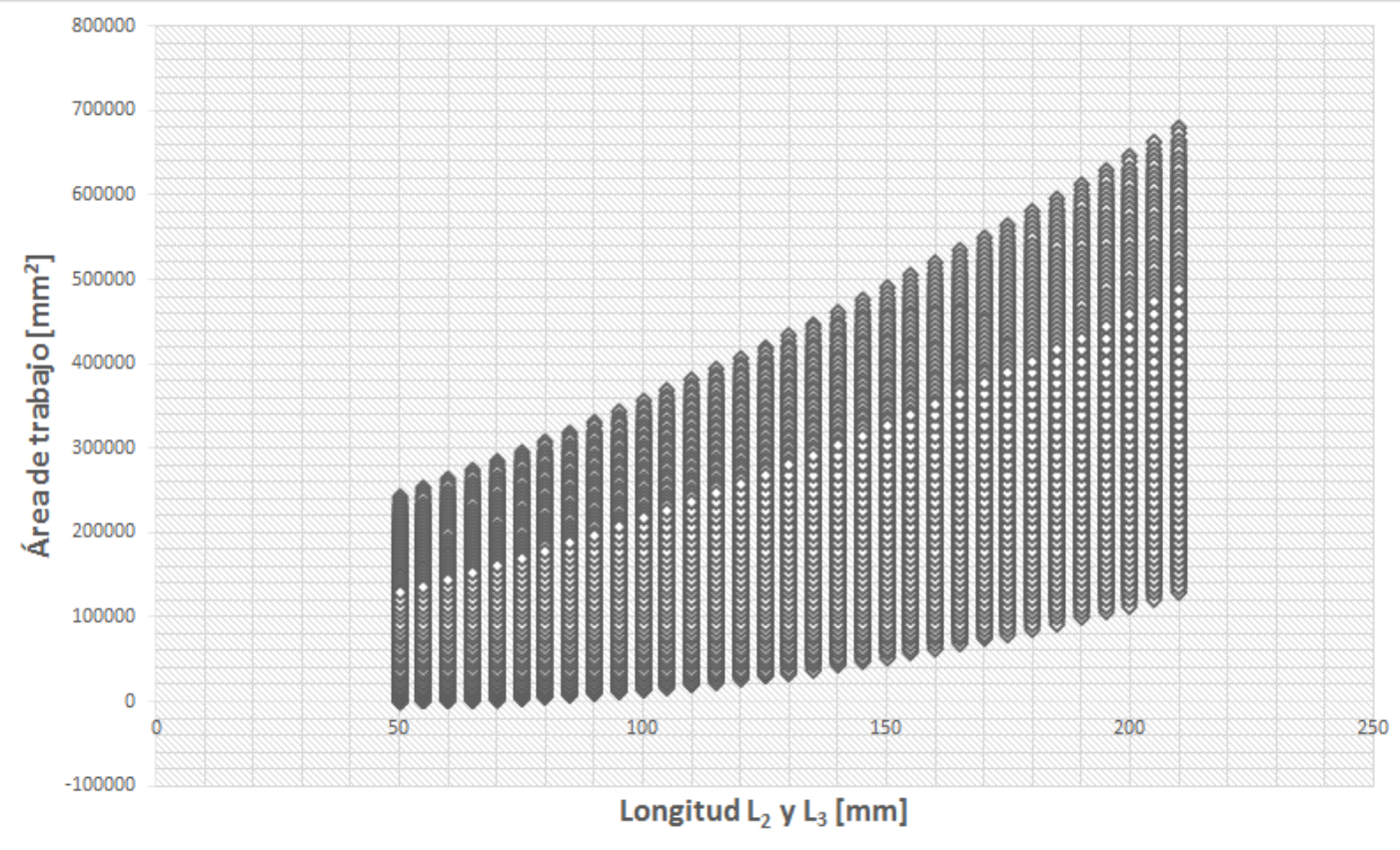

Fig. 5. $L_{2} y L_{3}$ vs. área de trabajo 


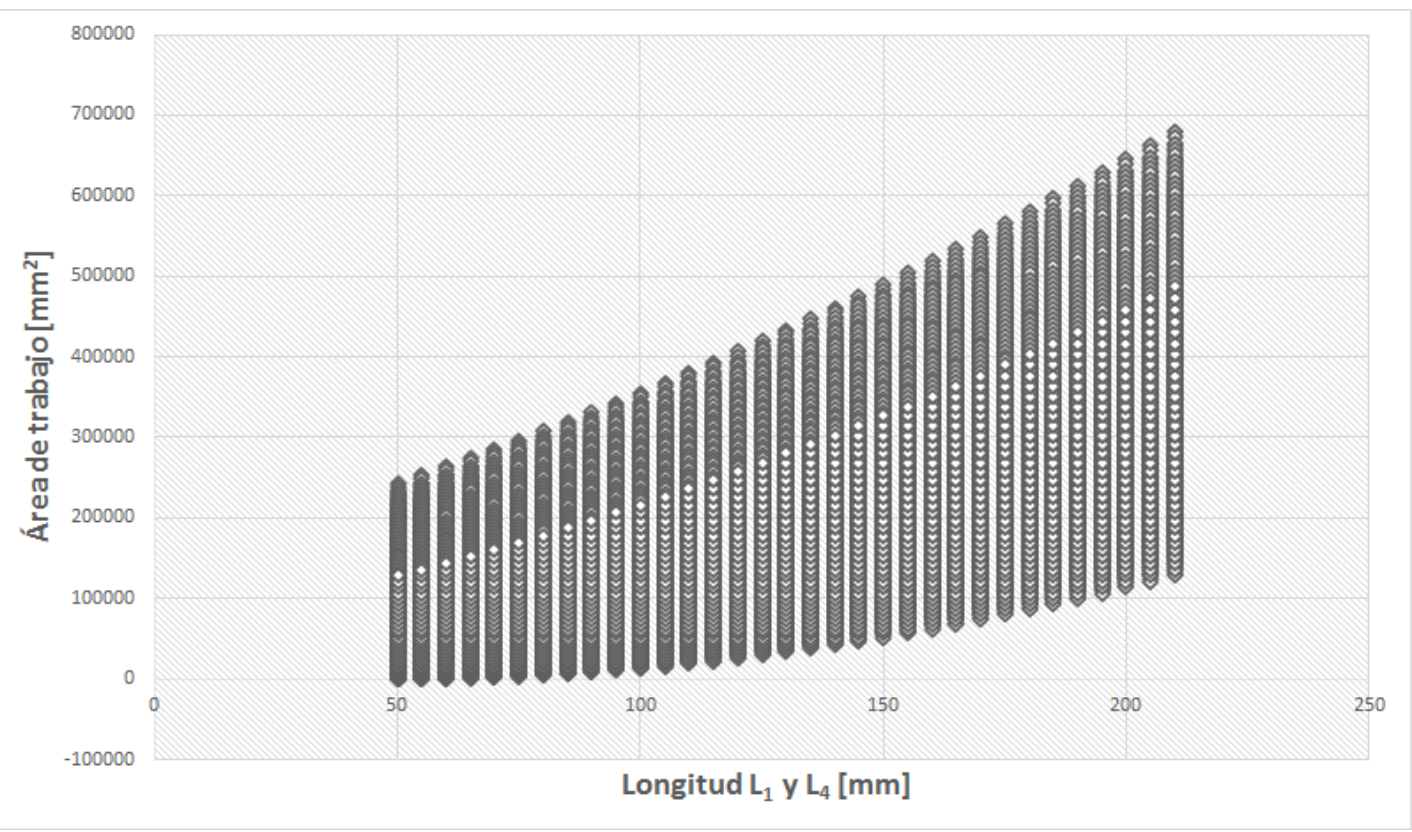

Fig. 6. $L_{1}$ y $L_{4}$ vs. área de trabajo

La Tabla 1 muestra la combinación con mayor área de trabajo calculada por el script, sin embargo, por restricciones físicas como el tamaño del efector final y tamaño de los actuadores, se elige la combinación 2. A pesar de que la combinación 2 tiene considerablemente menor área de trabajo que la combinación 1, esta permite cumplir con la tarea definida en la sección A y la trayectoria propuesta. Se verifica esto con el análisis de cinemática inversa calculado en la sección B. La región sombreada de la Fig. 7 y 8 muestran el área de trabajo que se obtiene con ambas combinaciones de la tabla y el rectángulo morado muestra el área meta propuesta para el script.

TABLA 1

DIMENSIONES DE LOS ESLABONES

\begin{tabular}{crrrrr}
\hline $\begin{array}{c}\text { Número de } \\
\text { combinación }\end{array}$ & $L_{0}(\mathrm{~mm})$ & \multicolumn{1}{c}{ Longitud } \\
\hline $1(\mathrm{~mm})$ & \multicolumn{1}{c}{$L_{2}(\mathrm{~mm})$} & $L_{3}(\mathrm{~mm})$ & $L_{4}(\mathrm{~mm})$ \\
\hline $\mathbf{1}$ & 70 & 175 & 175 & 210 & 210 \\
$\mathbf{2}$ & 150 & 135 & 135 & 210 & 210 \\
\hline
\end{tabular}




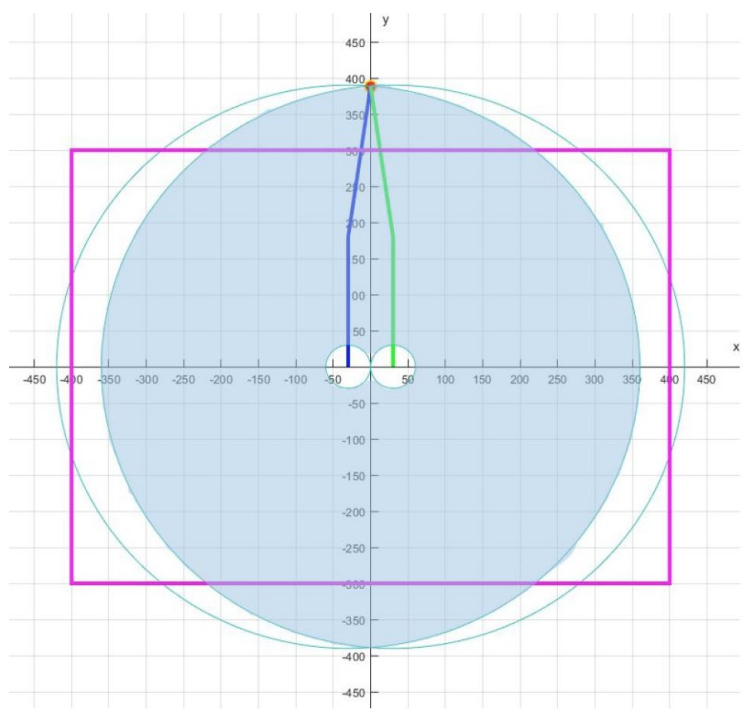

Fig. 7. Área de trabajo combinación número 1.

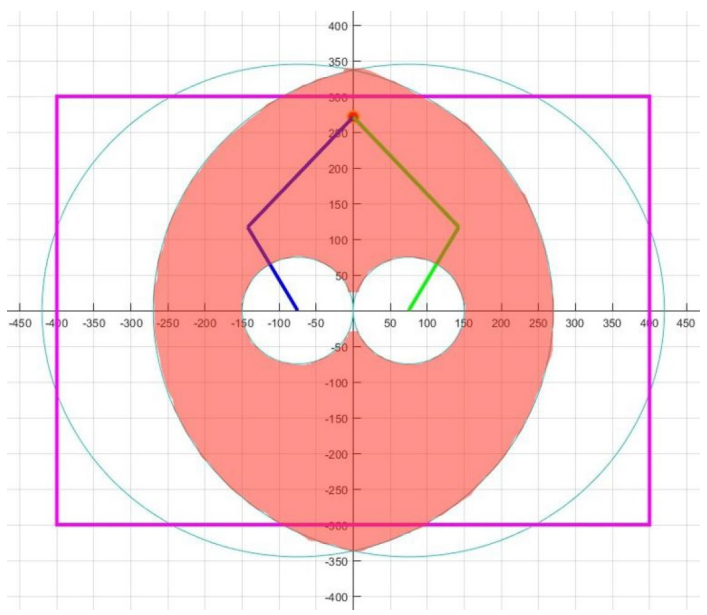

Fig. 8. Área de trabajo combinación número 2.

\section{D) Propuesta de actuadores}

Una vez definida el área de trabajo, el diseñador debe proponer un tipo de actuador rotacional para el robot tomando en cuenta el objetivo y las restricciones definidas en la sección A.

Se propone utilizar motores brushless (sin escobillas) por las ventajas que estos ofrecen, ya que, al no utilizar escobillas, tiene una mayor vida útil que un motor DC, de igual manera no genera ruido en la operación causado por arcos eléctricos, lo cual es común en motores de escobillas. Además, el motor brushless permite operar con carga nominal a altas velocidades [9]. El sistema está propuesto utilizando los motores brushless de manera directa, sin ningún tipo de reducción, para evitar la imprecisión que estas puedan presentar y así obtener la precisión definida en la sección A.

Se propone utilizar el motor YUNM4234 que cuenta con las siguientes especificaciones: resistencia interna $0.04 \Omega, 7$ par de polos y una constante de velocidad KV700 (rpm por volt). El motor se recoge con una baja 
resistencia interna para que se pueda utilizar una etapa de potencia de un voltaje menor, idealmente igual o menor a $12 \mathrm{~V}$. Entre mayor número de polos se puede tener un movimiento más suave y preciso, 7 es un número común para este tipo de motores. La constante KV va directamente ligada al par que puede entregar el motor (véase sección $\mathrm{K}$ ); generalmente entre menor es este número mayor es el par nominal pero la velocidad nominal es menor.

\section{E) Propuesta de material y características de eslabones.}

Con base a las restricciones definidas y las longitudes calculadas, el diseñador debe de proponer un material y un diseño para los eslabones. Para el robot se propone utilizar barra redonda de fibra de carbón de $1 / 4$ de pulgada de diámetro por su rigidez, bajo peso, durabilidad y bajo costo.

\section{F) Diseño $C A D$}

Una vez seleccionado el actuador y propuesto un material y forma de los eslabones (secciones D y E) se realiza un diseño CAD incluyendo el resto de los componentes mecánicos necesarios. El CAD debe ser muy preciso ya que funciona como la base de los análisis y simulaciones de las siguientes secciones. Utilizando SolidWorks se diseña un prototipo del robot, el cual puede ser visto en la Fig. 9. Los componentes mecánicos que se seleccionan son: Perfil de aluminio extruido $20 \times 20 \mathrm{~mm}$ (1640 mm de longitud total), impresión 3D para base de los motores y la unión de los eslabones, baleros M4 y tornillería M4. El volumen total de la estructura es de $2,707.5 \mathrm{~cm}^{3}$.

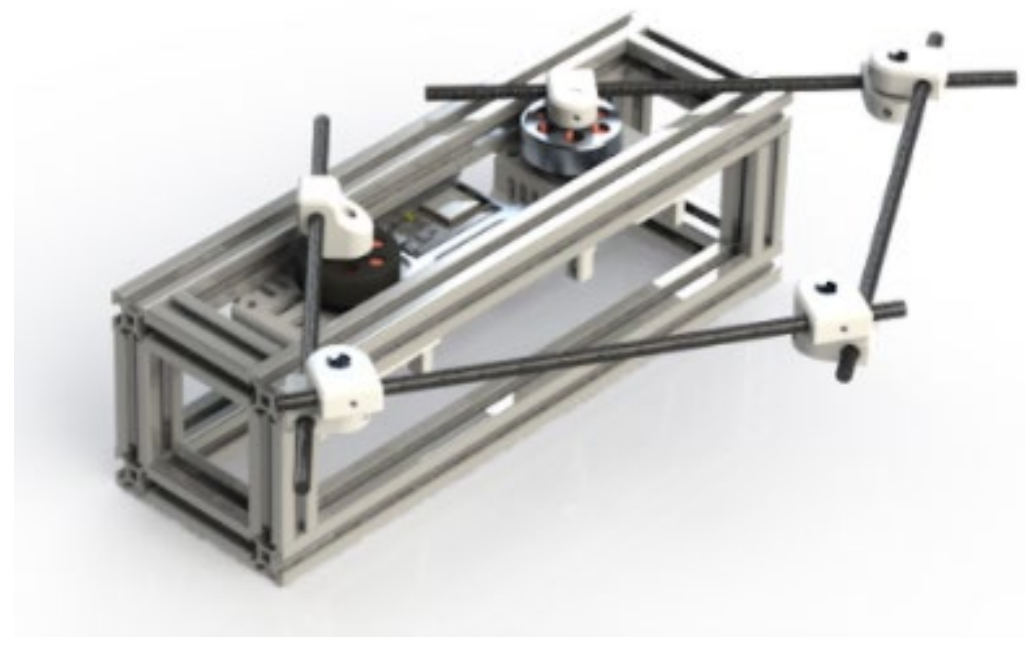

Fig. 9. Vista Isométrica superior $C A D$.

\section{G) Simulación dinámica}

Con ayuda de un software de simulación, como puede ser Matlab o Adams, el diseñador realiza una simulación dinámica que permite conocer el par máximo que requieren los actuadores en función del tiempo, al realizar la trayectoria planteada en la definición de problema (sección A). Aquí el diseñador debe incluir un controlador para el seguimiento de trayectoria durante la simulación asegurando que se cumplen las restricciones marcadas en la sección A. El diseñador debe observar que no existan picos de par en la gráfica al realizar la trayectoria planeada, ya que esto indicaría que la trayectoria del robot pasa por alguna singularidad. De existir un pico así 
se debe buscar si se puede realizar la misma trayectoria de manera diferente o planear una nueva trayectoria que logre cumplir con el mismo objetivo.

Se utiliza el software Adams para la simulación dinámica del robot. La Fig. 10 muestra el par máximo que requieren los actuadores para mover los eslabones con la trayectoria propuesta. El par máximo calculado fue de $100 \mathrm{Nmm}$, es decir, $0.1 \mathrm{Nm}$. Se incorpora un controlador PI de velocidad en cascada con un controlador $P$ de posición para el control de los actuadores.

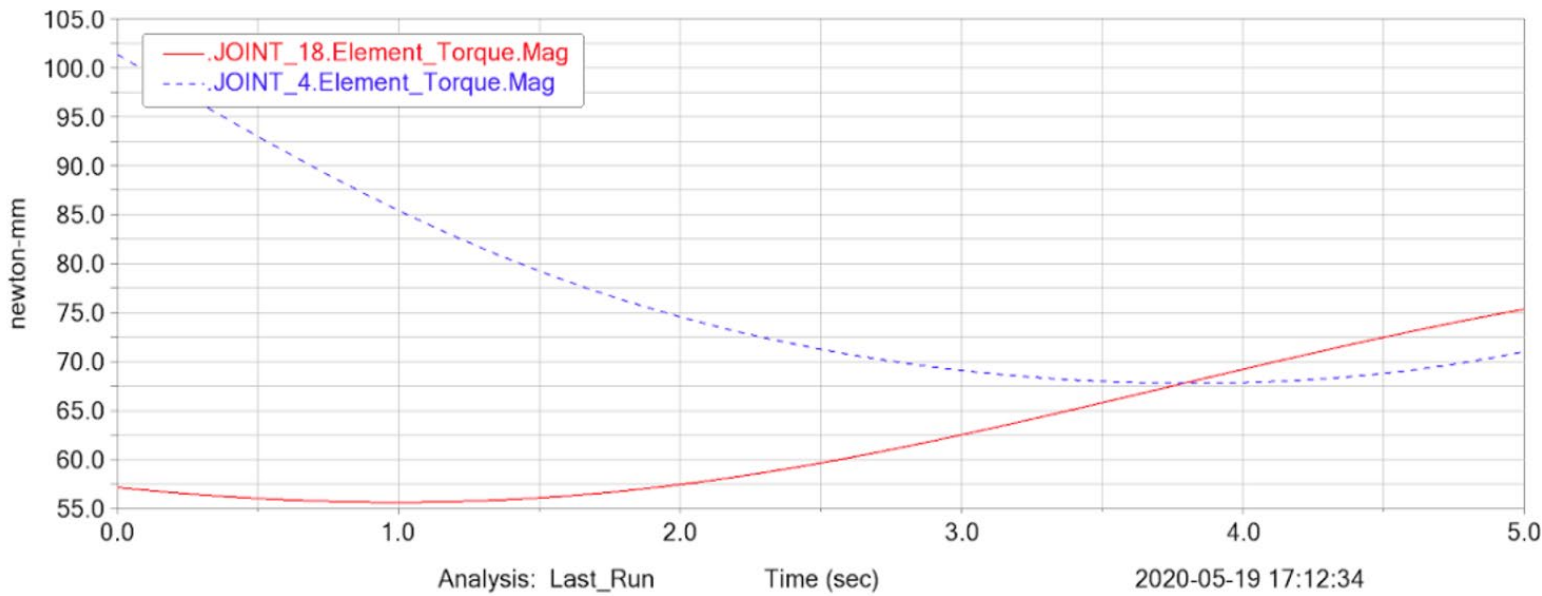

Fig. 10. Gráfica de par vs. Tiempo.

\section{H) Análisis por elemento finito}

El análisis por elemento finito permite al diseñador validar que los materiales y la geometría de su diseño es adecuada para llevar a cabo la tarea propuesta. El software Adams permite realizar un análisis por elemento finito de un CAD al proponer una trayectoria y al definir el material de los elementos. En la Fig. 11 se observa que la deformación máxima de los eslabones es de 3.45 E-03 mm.
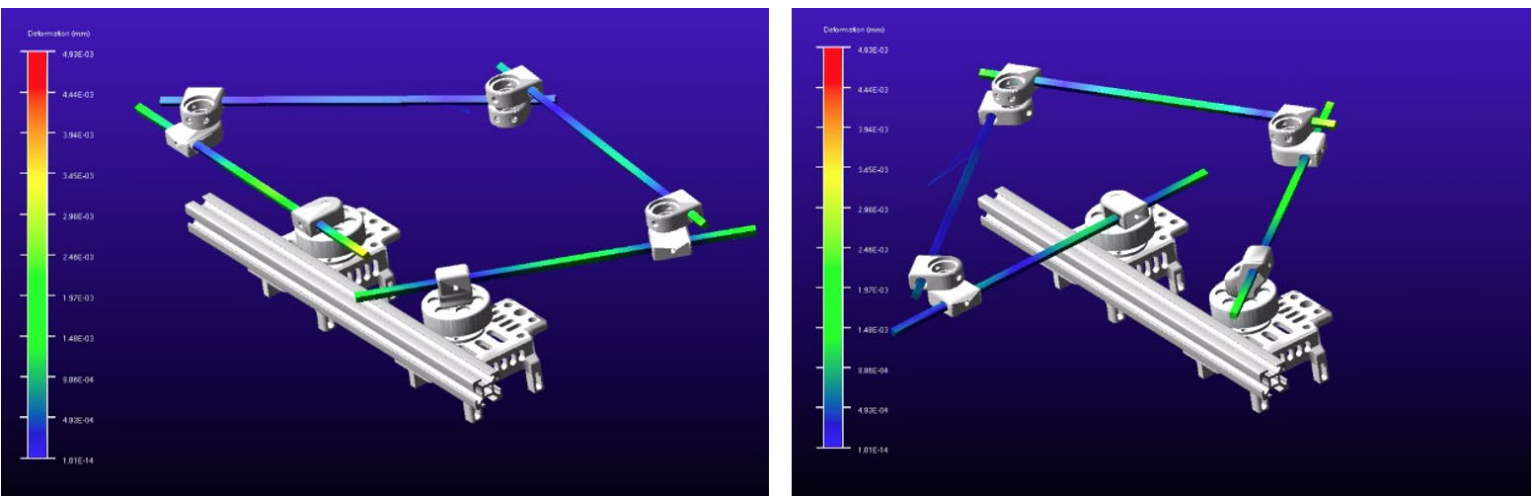

Fig. 11. Análisis de elemento finito posición media y final de trayectoria 
En caso de que la deformación sea inaceptable, se debe volver a proponer el grosor de los eslabones (sección E) y repetir los pasos anteriores hasta este punto, sección $\mathrm{F}$ a H.

\section{I) Construcción de prototipo}

El prototipo se construye una vez que los pasos (A-H) son validados con las simulaciones necesarias. La Fig. 12 muestra el prototipo armado del robot. Es importante que el prototipo físico sea lo más cercano al diseño CAD para poder tomar las simulaciones como válidas.

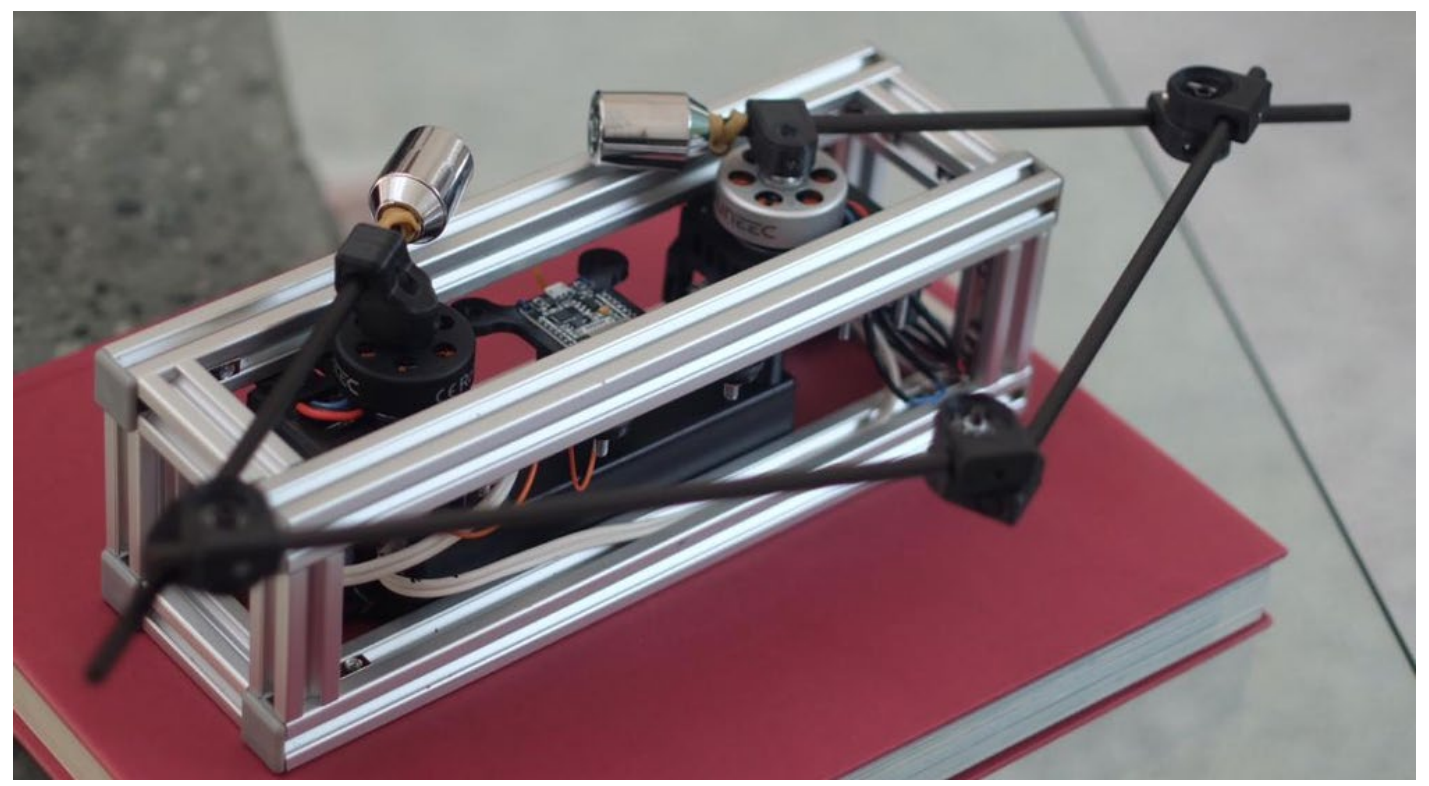

Fig. 12. Prototipo físico de robot

\section{J) Programación de microcontrolador}

El diseñador debe programar la trayectoria propuesta (sección A), calculada con cinemática inversa (sección B), para que realice la tarea definida en la problemática (sección A). Para esto el diseñador debe implementar el controlador de posición para los actuadores considerado en la simulación dinámica (sección G).

El controlador del robot está basado en el microcontrolador ESP32 programado en Arduino, utilizando la librería llamada SimpleFOC para el control de posición de los motores brushless [10]. Esta librería permite implementar el controlador PI de velocidad en cascada con el controlador P de posición propuesto en la sección G.

\section{K) Selección/diseño de etapa de potencia}

La etapa de potencia debe ser adecuada para el actuador/motor seleccionado y el par máximo requerido (secciones D y G). Esta puede ser escogida dentro de soluciones existentes o puede ser diseñada de manera específica para el diseño.

Como en la sección D se escoge utilizar motores brushless se deben considerar las siguientes ecuaciones para calcular la corriente máxima que debe soportar el controlador. La ecuación (16) muestra una manera de 
aproximar el par ideal para motores brushless dada una corriente. La ecuación (17) permite calcular la corriente necesaria para obtener el par máximo requerido, es decir, $0.1 \mathrm{Nm}$ (sección $\mathrm{G}$ ).

$$
\tau=\frac{60}{2 \pi} * \frac{i}{K V}
$$

$$
i=K V * \tau * \frac{2 \pi}{60}=700 * 0.1 * \frac{2 \pi}{60}=7.35[A]
$$

Con base a la corriente calculada se selecciona una etapa de potencia dentro de la especificación. Si la corriente calculada es mayor a la que puede soportar el motor, hay que regresar a la propuesta de actuador (sección D).

El control de los motores brushless se realiza utilizando la tarjeta desarrollada llamada JANUS CONTROLLER 20.01 (véase Fig. 13). Esta tarjeta implementa el algoritmo de Field Oriented Control (FOC) para el control de posición y velocidad de motores brushless. La tarjeta tiene tres elementos principales: una etapa de potencia basada en MOSFETS, un sensor de temperatura y un encoder magnético con una resolución de 0.088 grados.

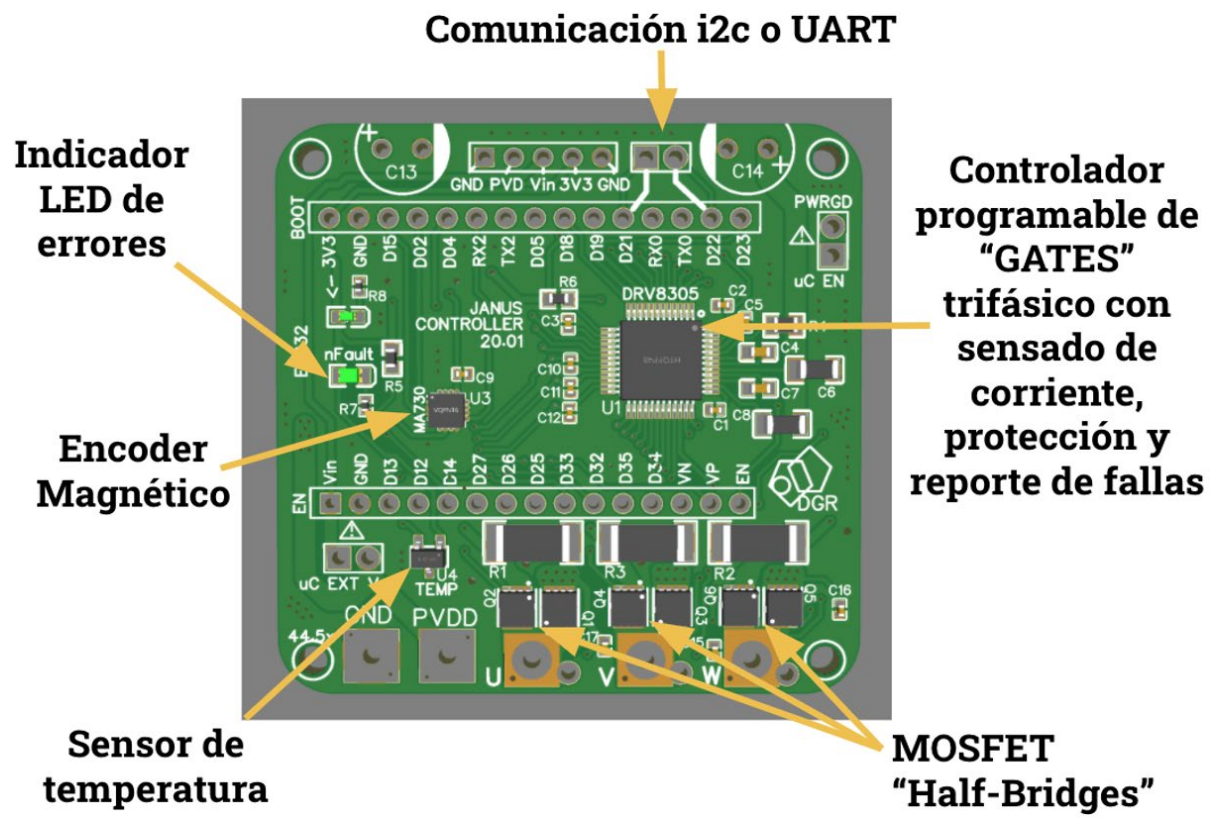

Fig. 13. Elementos principales del JANUS CONTROLLER 20.01

\section{L) Diseño de interfaz (opcional)}

El diseñador tiene la opción de diseñar una interfaz la cual permita a un operador reproducir secuencias programadas, programar nuevas secuencias o controlar directamente el efector final. Para el robot se implementa una interfaz humano-máquina que permite a un operador mover el efector final del robot al dar instrucciones a través de un joystick virtual (resolviendo cinemática inversa, sección B). Además, incluye 
botones para reproducir trayectorias preprogramadas y puede salvar y reproducir trayectorias programadas por el operador (véase Fig. 14).

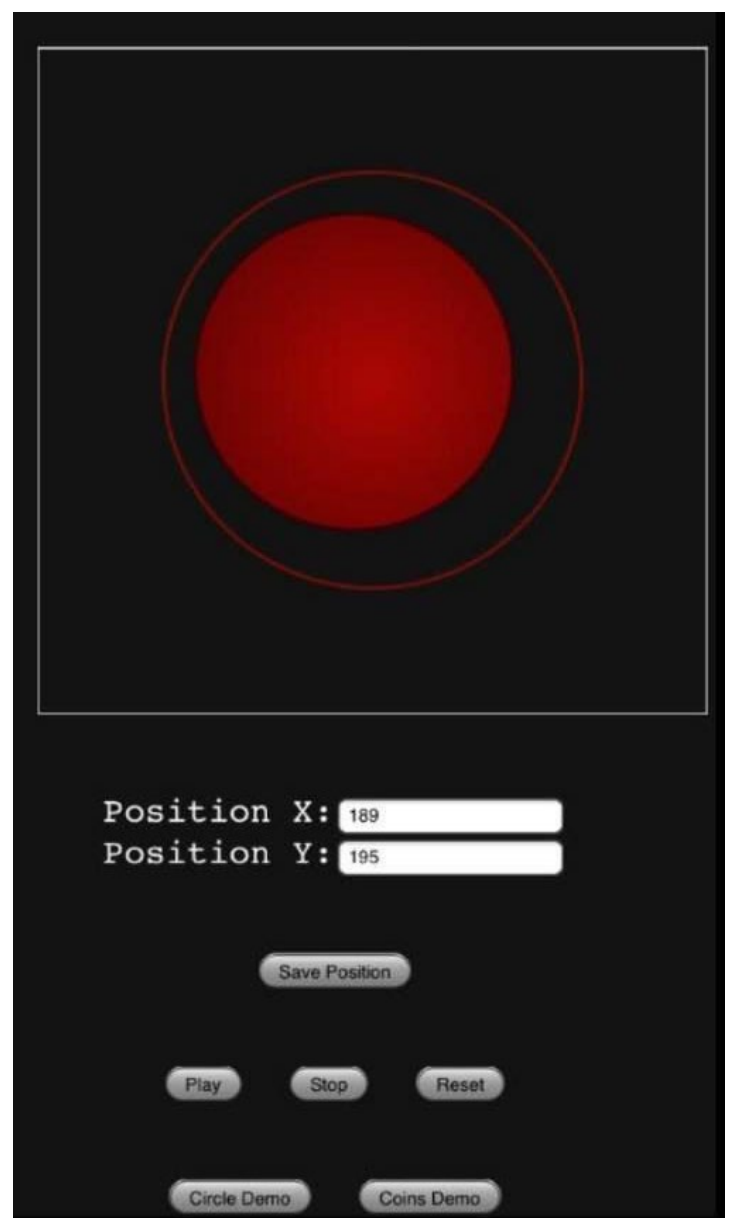

Fig. 14. Interfaz HMl robot

\section{RESULTADOS}

El robot cumple satisfactoriamente con la tarea propuesta moviendo una masa de 100 gramos a una velocidad de hasta $1 \mathrm{~m} / \mathrm{s}$. Tiene un área de trabajo de $340101.1927 \mathrm{~mm}^{2}$, precisión para tareas de pick and place de $\pm 4 \mathrm{~mm}$ (medido experimentalmente) y una masa total del robot de $1650 \mathrm{~g}$.

La validación del controlador se realiza al reproducir una trayectoria circular del efector final. Se proponen dos velocidades de operación, dos y cuatro segundos respectivamente, donde se mide el error obtenido en el seguimiento sin la modificación de los parámetros del controlador. Se grafica en el plano la posición experimental del efector final contra la posición teórica, como se observa en las Figs. 15 y 16, donde se observa el error porcentual contra el tiempo. El error promedio del primer intervalo de tiempo es de $9.8658 \%$, el segundo es del $4.02 \%$. El error de seguimiento es menor al $5 \%$ en la trayectoria de 4 segundos, por lo que se considera aceptable para trabajos de baja velocidad. 


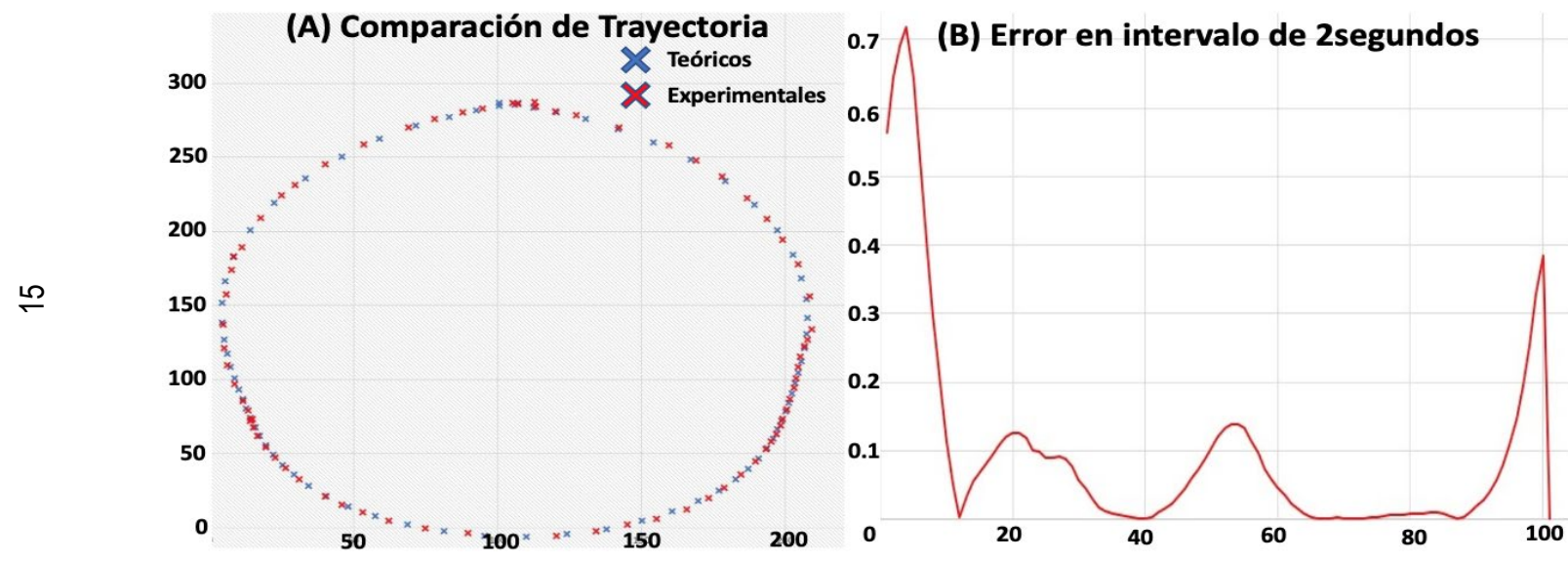

Fig. 15. Seguimiento de trayectoria (A) y error (B) en intervalo de 2 segundos.

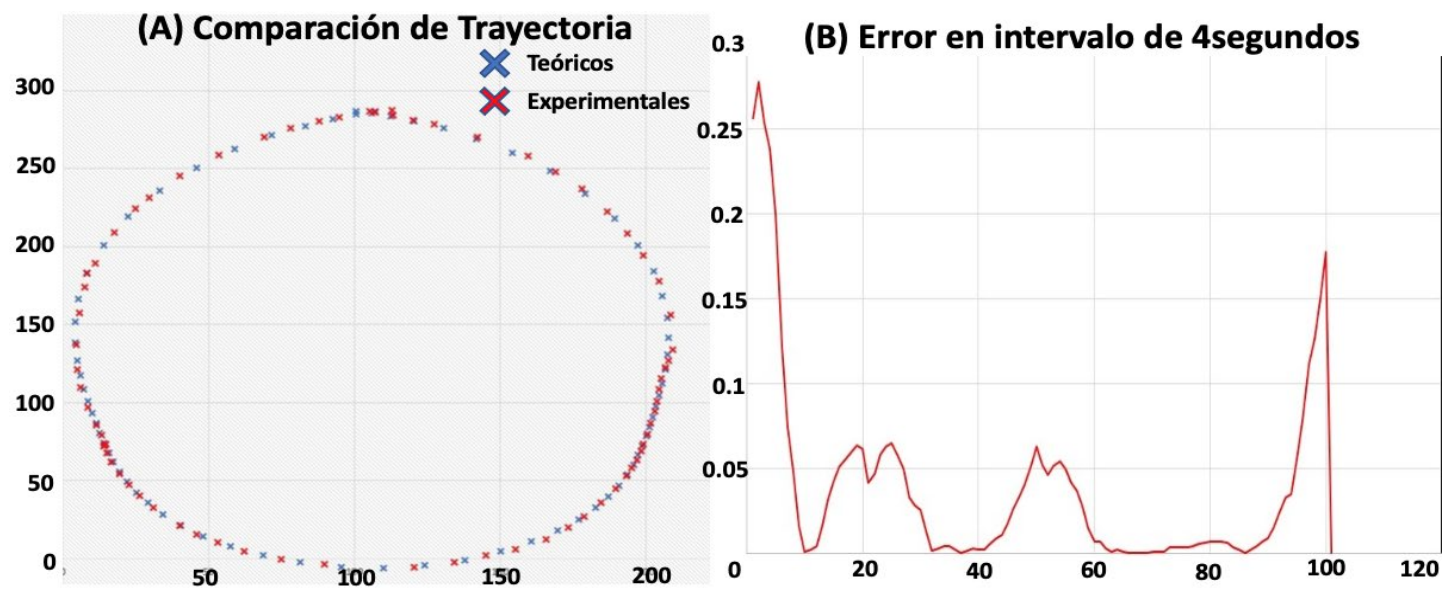

Fig. 16. Seguimiento de trayectoria $(A)$ y error $(B)$ en intervalo de 4 segundos

\section{CONCLUSIONES}

La metodología creada es útil si no se conoce el modelo dinámico del robot, ya que este proceso solamente utiliza síntesis estructural y dinámica no lineal del sistema. Esto hace que la metodología sea accesible, ya que no se tiene problema con el modelo dinámico y todos los pasos que lo requieren son sustituidos por simulaciones, las cuales representan los requerimientos del sistema. Esto permite al diseñador agilizar el proceso de diseño y permitir ajustes en el diseño de manera eficiente.

Se demuestra que el control de posición de motores brushless se puede hacer sin necesidad de equipo industrial y que se pueden aplicar para la robótica y la automatización. Es importante seleccionar el motor brushless adecuado y para esto se debe conocer el par requerido.

El robot paralelo de cinco eslabones y dos grados de libertad tiene beneficios y desventajas, por lo que hay que conocer la aplicación para saber si es el brazo robot adecuado. Entre las ventajas del robot está la 
adaptabilidad mediante la selección de las longitudes de los eslabones y la repartición de esfuerzo entre los actuadores. En el cálculo de longitudes de los eslabones se observa que aumentar la longitud del eslabón $L_{0}$ afecta negativamente el área total de trabajo mientras que incrementar la longitud de los demás eslabones la afecta positivamente.

\section{REFERENCIAS}

[1] J. Martínez-Frutos, P. Martí, Diseño óptimo robusto utilizando modelos Kriging: aplicación al diseño óptimo robusto de estructuras articuladas. Revista Internacional de Métodos Numéricos para Cálculo y Diseño en Ingeniería, vol. 30, no. 2, 2014, pp. 97-105, doi: https://doi.org/10.1016/j.rimni.2013.01.003

[2] Ben Hamida, M. A. Laribi, A. Mlika, L. Romdhane, S. Zeghloul, G. Carbone, Multi-Objective Optimal Design of a Cable Driven Parallel Robot for Rehabilitation Tasks, Mechanism and Machine Theory, vol. 156, 2021, doi: https://doi.org/10.1016/j.mechmachtheory.2020.104141

[3] E. H. Arias-Nava, A. J. Ríos-Lira, J. A. Vázquez-López, R. Pérez-González, Estudio comparativo entre los enfoques de diseño experimental robusto de Taguchi y tradicional en presencia de interacciones de control por control, Ingeniería, Investigación y Tecnología, vol. 16, no. 1, 2015, pp. 131-142, doi: https://doi.org/10.1016/S1405-7743(15)72114-1

[4] M. Aquino, E. Pozo, O. Zambrano, W. Venegas, Análisis cinemático y dinámico de un robot paralelo según el principio de trabajos virtuales para rehabilitación, Proyecto PIMI 15-04: Robot Paralelo, 2017.

[5] P. Shao, Z. Wang, S. Yang, Z. Liu, Dynamic modeling of a two-DoF rotational parallel robot with changeable rotational axes, Mechanism and Machine Theory, vol. 131, 2019, pp. 318-335, doi: https://doi.org/10.1016/j.mechmachtheory.2018.08.020

[6] Cammarata, I. Caliò, D. D’Urso, A. Greco, M. Lacagnina, G. Fichera, Dynamic stiffness model of spherical parallel robots, Journal of Sound and Vibration, vol. 384, 2016, pp. 312-324, https://doi.org/10.1016/j.jsv.2016.08.014

[7] M. Díaz, S. Provenzano, M. Vergara, R. Chacón, Dinámica Directa de Robots Paralelos Utilizando las Ecuaciones de Gibbs-Appell, Información Tecnológica, vol. 18, no. 4, pp. 75-82, 2007, doi: http://dx.doi.org/10.4067/S071807642007000400011

[8] L. Tien, K. Hee-Jun, D. Quang, A method for optimal kinematic design of five-bar planar parallel manipulators. 2013 International Conference on Control, Automation and Information Sciences, ICCAIS 2013, pp. 7-11, doi: https://doi.org/10.1109/ICCAIS.2013.6720521

[9] R. Crowder, Brushless motors, Electric Drives and Electromechanical Systems, 2nd ed., Butterworth-Heinemann, 2020, pp. 165-185.

[10] A. Skuric, Arduino Compatible Open Source Field Oriented Control (FOC) project, SimpleFOCproject, 2020 https://simplefoc.com/ 